\title{
Caractérisation des unités de séchage des ananas et qualités physicochimiques des produits alimentaires dérivés dans la région du Littoral Cameroun
}

\author{
Mara Naomie NGO BOGMIS ${ }^{1}$, Gérard NGOH NEWILAH ${ }^{2,3 *}$ et \\ Robert NDJOUENKEU ${ }^{4}$ \\ ${ }^{1}$ Institut de Recherche Agricole pour le Développement (IRAD) Programme fruits BP 13 Njombé. \\ ${ }^{2}$ Département de Biochimie, Université de Dschang, BP 67 Dschang - Cameroun. \\ ${ }^{3}$ Laboratoire de Technologie Post-Récolte, Centre Africain de Recherches sur Bananiers et Plantains, BP 832 \\ Douala, Cameroun. \\ ${ }^{4}$ Ecole Nationale Supérieure des Sciences Agro Industrielles, Université de Ngaoundéré, BP 454 Ngaoundéré- \\ Cameroun. \\ *Corresponding author; E-mail: gbngoh@gmail.com ; Tel: 00237679477555
}

\section{RESUME}

Une étude de caractérisation des unités de séchage et des fruits séchés a été effectuée dans la région du Littoral - Cameroun. Son objectif était de faire un diagnostic des structures de séchage et une évaluation des qualités physicochimiques des ananas séchés en vue de dégager les points d'amélioration technologique et d'appui technique aux acteurs. Au Cameroun, le séchage de l'ananas est semi-artisanal et la méthodologie diffère selon le transformateur. 35 échantillons d'ananas séchés ont été collectés dans 7 unités en activité des 10 unités de séchage enquêtées selon une approche participative pour leurs caractéristiques et leur fonctionnement. Les paramètres suivants ont été analysés: teneur en eau résiduelle, teneur en matière sèche, taux d'extrait sec soluble, $\mathrm{pH}$, acidité titrable et teneur en sucres totaux. Les résultats ont montré une différence significative $(\mathrm{p}<0,05)$ entre les productions; les ananas séchés obtenus étaient de qualités physicochimiques moins satisfaisantes. La teneur en eau dans la plupart des produits séchés était relativement plus élevée que $15 \%$. Quatre échantillons sur trente-cinq ont présenté un taux d'extrait sec conforme à la norme. De plus, l'enquête a révélé des contraintes liées aux matières premières et aux procédures de séchage. L'optimisation d'un protocole standard et l'acquisition d'équipements appropriés seraient importants pour le développement de cette activité.

() 2016 International Formulae Group. All rights reserved.

Mots clés : Unité de séchage, propriétés physicochimiques, ananas séchés, Littoral-Cameroun.

\section{Characterization of pineapples drying units and physicochemical qualities of derived food products in Cameroon}

\begin{abstract}
This study based on the characterisation of drying units and dried fruits was carried out in Cameroon (Littoral region). The purpose was to establish a diagnosis of the drying units available and assess
\end{abstract}


physicochemical properties of dried pineapples in order to identify technical and technological gaps to be improved. In Cameroon, pineapple drying process is semi-industrial and methodology differs according to processors. Through a participatory approach, 35 dried pineapple samples were collected in 7 units in activity of the 10 drying units investigated for their characteristics and functioning. They were analyzed for the following parameters: residual water content, dry matter content, total soluble solids extracts, $\mathrm{pH}$, titratable acidity and total sugar content. The results showed significant differences $(p<0.05)$ between dried pineapples that were of poor physicochemical qualities. The water content in most dried products was relatively higher than $15 \%$. Only four of the thirty-five samples collected presented compliant total soluble solid extract contents. The investigation revealed the constraints related to raw materials and drying procedures. Optimizing a standard protocol and the acquisition of appropriate drying equipment would be important for the development of this activity as well as the improvement of the quality of dried pineapple.

(C) 2016 International Formulae Group. All rights reserved.

Keywords: Drying unit, physicochemical properties, dried pineapple, Littoral-Cameroon.

\section{INTRODUCTION}

L'ananas (Ananas comosus) est une plante de la famille des broméliacées qui présente un intérêt économique indéniable au Cameroun. Sa production est estimée à environ 168000 tonnes sur une superficie cultivée de 4800 ha soit 35 tonnes /ha (FAOSTAT, 2014). L'ananas occupe après la banane, la deuxième place des fruits à l'exportation vers les marchés de l'Union Européenne (AGROCOM, 2002). Cinq cultivars d'ananas décrits par de nombreux travaux antérieurs réalisés par le CIRAD en 2008, et rapportés par Tossou et al. (2014) sont habituellement rencontrés dans le monde en général et au Cameroun en particulier, où ils sont cultivés principalement dans 7 régions méridionales (Est, Sud, Centre, Ouest, Nordouest, Sud-ouest et Littoral) des 10 régions du pays. Pour répondre aux exigences commerciales, les producteurs camerounais cultivent 3 variétés (Cayenne lisse, Queen, Red spanish) qui ont été diffusées à travers le pays après de nombreux travaux de recherche (AGROCOM, 2002). La consommation du fruit se fait sous sa forme native ou sous forme transformée. Dans ce dernier cas, les principales formes de transformation sont le jus, la confiture, les lamelles ou les tranches séchées. En raison de sa forte teneur en eau qui représente 80 à $90 \%$ de son poids (Brat et al., 2004), l'ananas est un produit fragile dont la conservation en frais est délicate. Le degré de périssabilité élevé du fruit pousse à une vente accélérée à la récolte, ce qui limite les possibilités d'accroissement des revenus des producteurs. Il arrive souvent, pour des producteurs avisés, que les fruits non vendus soient transformés en jus et confiture, ou encore séchés en tranches cylindriques.

Le séchage est un moyen de conservation utilisé pour fabriquer de nouveaux produits. Très souvent, les producteurs manquent de connaissances techniques sur le fruit à sécher et travaillent de façon particulière, sans cadre de concertation qui permettrait d'améliorer la qualité et la rentabilité de leurs activités. La conséquence est une variabilité des pratiques et de la qualité entre les acteurs et même entre les productions d'une même unité. En ce qui concerne les fruits séchés au Cameroun, Tchango Tchango (2003) a identifié une quinzaine d'unités locales de séchage des fruits dans les régions de l'Ouest et du Littoral. Dans ces unités, l'ananas comme la plupart des fruits séchés, fait l'objet d'un commerce international intéressant dans la mesure où plus de $80 \%$ des fruits séchés sont destinés à l'exportation (Hamadé, 2003). Cependant, les résultats obtenus par AGROPME (2003) ont mis en évidence des problèmes de qualité des produits qui portent entre autres sur: la texture collante, la valeur gustative, la qualité de l'emballage limitant leur acceptation par le marché. De plus, des études locales menées par l'Institut de Recherche Agricole pour le Développement 
(IRAD) sur le séchage des fruits, montrent que la majorité de ces produits n'opère pas de percée significative dans la consommation nationale des ménages (Ndindeng, 2005). Par ailleurs, dans ces unités de transformations, les pratiques sont généralement fondées sur la base d'acquis technologiques traditionnels, avec des procédés de séchage semi-artisanaux. Il s'avère important de décrire les procédures utilisées pour la production des ananas séchés au Cameroun. L'objectif de cette étude a été d'identifier et de caractériser les unités de séchage produisant les ananas séchés dans la région du Littoral, et d'évaluer les qualités physicochimiques des échantillons collectés dans chaque unité en vue de dégager les points et orientations d'amélioration technologique des pratiques existantes.

\section{MATERIEL ET METHODES}

\section{Diagnostic des unités de séchage}

Cette étude a été réalisée de Mars à Août 2009 dans dix unités de séchage de la région du Littoral au Cameroun. Les structures visitées étaient situées dans deux départements de cette région : le Wouri et le Moungo. La zone urbaine de Douala a été choisie à cause de l'existence de grands marchés de fruits et des structures de transformation en rapport avec les facilités de commercialisation. Le département du Moungo a été retenu dans cette étude parce qu'il abrite des grands bassins de production des fruits et légumes dans les localités de Njombé, Penja et Loum.

Une enquête selon une approche participative a été réalisée auprès des transformateurs dans les unités visitées. Les informations étaient recueillies au cours des visites de structures, des entretiens avec les responsables et membres des groupes. Sur la base d'un questionnaire élaboré selon la méthode d'interview semi structuré, les données collectées concernaient la typologie des unités et le procédé de séchage de l'ananas. Ces informations ressortaient le fonctionnement des unités, la gestion des fruits frais, les différentes étapes de transformation partant de l'achat ou de la récolte du fruit jusqu'à la commercialisation des produits séchés en passant par les procédés de séchage appliqués par les opérateurs, les méthodes de stockage et de contrôles effectués sur les fruits frais et les produits séchés.

\section{Détermination des paramètres physicochimiques des fruits séchés}

La caractérisation physicochimique des produits séchés collectés dans les unités de transformation a été réalisée au Laboratoire de Technologie Post-Récolte (TPR) du Centre Africain de Recherches sur Bananiers et Plantains (CARBAP) basé à Njombé au Cameroun. En effet, les échantillons de fruits séchés ont été prélevés parmi les sachets de 50 $\mathrm{g}$ et $100 \mathrm{~g}$ mis en vente à la fin de chaque production dans les sept unités qui étaient en activité pendant l'étude; il s'agissait des unités suivantes: C1, U2 et M3 dans le département du Moungo et T4, J5, Z6 et B7 dans le département du Wouri. Ces échantillons ont été analysés pour les paramètres suivants : teneur en eau, acidité totale titrable, extrait sec soluble, $\mathrm{pH}$, et sucres totaux.

\section{Teneur en eau et en matière sèche}

La teneur en eau a été déterminée selon la méthode décrite par l'AFNOR (1981) et appliquée par Agassounon et al. (2012). La détermination a été faite à $105^{\circ} \mathrm{C}$ pendant 24 heures jusqu'à l'obtention d'un poids constant. Les résultats présentés sont les moyennes \pm écarts types de trois déterminations. La teneur en eau (TE) a été exprimée en g d'eau pour $100 \mathrm{~g}$ de produit séché et calculée selon la formule ci-après :

$$
\mathrm{TE}=\left[\left(\mathrm{M}_{1}-\mathrm{M}_{2}\right) /\left(\mathrm{M}_{1}-\mathrm{M}_{0}\right)\right] \times 100
$$

Où : M0 est la masse de la capsule vide; M1 est la masse de la capsule avec l'échantillon avant l'entrée à l'étuve; M2 est la masse de la capsule à la sortie de l'étuve.

La teneur en matière sèche (TMS) a été obtenue à partir de la relation précédente relative à la teneur en eau. Elle a été exprimée en $\mathrm{g}$ de matière pour $100 \mathrm{~g}$ de produit séché selon la formule suivante : TMS $=100-\mathrm{TE}$ 


\section{Acidité totale titrable}

L'acidité totale titrable a été dosée selon la méthode décrite par l'AFNOR (1982) sur les produits dérivés des fruits et légumes. La pulpe d'ananas séché (15 g) a été trempée dans $90 \mathrm{ml}$ d'eau distillée pendant 30 à 45 minutes, elle a été ensuite broyée dans un mixeur de cuisine (Moulinex de type AAW5 $220-240 \mathrm{~V} ; 50-60 \mathrm{~Hz}$ ) puis filtrée à l'aide d'un papier filtre (Whatman $\mathrm{n}^{\circ} 1$ ). La neutralisation de l'acide avec la solution de soude a été opérée jusqu'à l'obtention d'une couleur rose persistante pendant 30 secondes. La descente de la burette a été notée et le volume en $\mathrm{ml}$ a été enregistré. L'Acidité Totale Titrable (ATT) exprimée en milliéquivalent pour $100 \mathrm{~g}$ de produit sec a été calculée selon la formule :

\section{$\left(100 / V_{0}\right)$}

$$
\mathrm{AT}=(\mathbf{9 0} / \mathrm{M}) \times f \times\left(\mathrm{V}_{1} / 10\right) \times
$$

Où : $\mathrm{f}$ est le facteur de dilution; $\mathrm{M}$ est la masse de l'échantillon en $g ; V_{0}$ est le volume (ml) de la prise d'essai; $\mathrm{V}_{1}$ est le volume (ml) de la soude noté au point de virage.

\section{Extrait sec soluble}

La détermination de l'extrait sec soluble a été réalisée selon Dadzie et Orchard (1997) à l'aide d'un réfractomètre. Une goutte de filtrat a été déposée sur le prisme d'un réfractomètre (Atago N-20, modèle $\mathrm{N}$, McCormick fruit Tech) qui a été ensuite pointé en direction d'une source lumineuse pour la lecture du degré Brix correspondant au taux d'extrait sec de l'échantillon. La valeur lue a été multipliée par le facteur de dilution de cette solution. La teneur en résidu sec soluble de la solution diluée a été exprimée selon la formule $\mathbf{I}_{\mathrm{R}}=\mathbf{M} \mathbf{x} \boldsymbol{f}$ où $\mathbf{M}$ est la valeur lue au réfractomètre et $f$ est le facteur de dilution.

pH

Le $\mathrm{pH}$ a été mesuré directement à l'aide des électrodes initialement étalonnées du pHmètre (Inolab, pH level 2). La valeur du $\mathrm{pH}$ a été enregistrée quelques instants après la stabilisation de la mesure.

\section{Sucres totaux}

Les sucres totaux ont été dosés par spectrophotométrie $(\lambda=490 \quad \mathrm{~nm})$ selon la méthode décrite par Dubois et al. (1965). La quantité de glucose des échantillons a été déterminée à partir d'une gamme d'étalonnage qui donne une équation de la droite de régression de la forme $\mathrm{DO}=\mathrm{ax}+\mathrm{b}$ avec un coefficient de régression $\mathrm{R}^{2}$; où $\mathrm{a}, \mathrm{b}$ et $\mathrm{DO}$ sont connus; $x$ est la quantité inconnue de sucre contenue dans la solution d'essai.

\section{Analyses statistiques}

Les analyses statistiques ont été effectuées à l'aide du logiciel SPSS version 12.0. Le dispositif expérimental fait appel à un schéma de $7 \times 5$ ( 7 unités de séchage, 5 productions) et les caractéristiques physicochimiques des échantillons sont des variables. Une analyse des variances (ANOVA) a été réalisée. Les résultats des paramètres physicochimiques exprimés en moyenne \pm écart type ont été obtenus sur la base de trois (03) déterminations. Le test de DUNCAN a permis de classer les moyennes des échantillons pour voir s'il existe une différence significative entre les unités mais aussi entre les productions dans la même structure de séchage.

\section{RESULTATS}

Typologie des unités de séchage

Fonctionnement des unités de séchage

Dix (10) structures de transformation ont été identifiées et visitées dont six (06) et quatre (04) dans les départements du Wouri et du Moungo respectivement. Elles ont été crées par des camerounais entre 1993 et 2007 comme Petite et Moyenne Entreprise (PME) ou comme groupe d'initiative commune (GIC). Chaque unité emploie 3 à 15 personnes qui sont dans la plupart des cas des femmes. L'activité principale dans la majorité des unités est le séchage des fruits et légumes. Les fruits sont traités dans les ateliers et/ou hangars des domiciles utilisés comme unité de séchage.

\section{Motivations des transformateurs}

La Figure 1 présente les tranches d'âges des acteurs, les résultats obtenus montrent que $60 \%$ des personnes rencontrées étaient âgés de 30 à 40 ans. Le 
niveau d'instruction des responsables variait du primaire (30\%) au supérieur $(40 \%)$ avec un taux de $30 \%$ pour le secondaire. Les motivations de création des unités de séchage de l'ananas dans la région du Littoral sont multiples et variées, elles sont présentées dans la Figure 2. En effet, les acteurs justifient l'existence des structures de production d'ananas séchés par plusieurs raisons dont les plus importantes sont: l'augmentation de la valeur ajoutée, la diversification des revenus et la limitation des pertes des produits frais non commercialisés.

\section{Critères de choix de la matière première}

En général, les fruits d'ananas transformés sont issus des marchés locaux ou des parcelles paysannes proches de l'unité de transformation. La maturité, la fermeté et la fraîcheur du fruit constituent les principales caractéristiques qui guident le choix des ananas aptes au processus de séchage. Le choix de la matière première peut aussi se faire suivant les critères suivants : l'itinéraire technique de production (mettant en exergue l'utilisation ou non des produits chimiques pour l'entretien, le suivi, la lutte contre les maladies et ravageurs, etc.), la forme et le poids du fruit, l'épaisseur de la pulpe et la variété (Figure 3).

\section{Limites des unités liées à la production des} fruits séchés

Les difficultés que rencontrent les opérateurs par rapport à leur activité de séchage des ananas s'articulent autour de plusieurs points et dépendent des types d'entreprises. Il s'agit entres autres de l'approvisionnement en matière première, des locaux réservés au séchage, de l'équipement de séchage et du matériel utilisé lors du processus. Ces aspects sont ceux qui ont été régulièrement relevés par les transformateurs durant l'enquête.

\section{Processus de transformation de l'ananas}

Les unités visitées ont des étapes de travail plus ou moins semblables. La Figure 4 présente un résumé schématique de la procédure de séchage d'ananas composé de quatre opérations principales. Les opérations préliminaires et la préparation du fruit au séchage sont effectives dans toutes les unités. Quant au séchage, il présente des différences à plusieurs niveaux dans les structures de transformation. Le conditionnement des fruits séchés d'ananas est effectué dans des sachets de polyéthylène dont la qualité dépend des moyens mis par les acteurs dans l'achat de l'emballage.

\section{Limites des unités dans la commercialisation des fruits séchés}

Les problèmes de commercialisation se posent dans toutes les unités de séchage. Trois niveaux de vente ont été évoqués par les transformateurs, il s'agit du marché local, du marché national et du marché international. Pour les transformateurs travaillant en étroite collaboration avec leurs partenaires résidant à l'étranger, un rapport de vente est envoyé aux expéditeurs sur la qualité des produits reçus, les réclamations et les améliorations à apporter lors des prochaines livraisons.

\section{Caractéristiques physicochimiques Teneur en eau}

Les valeurs consignées dans le Tableau 1 représentent les moyennes de 3 déterminations obtenues après l'analyse des échantillons prélevés dans les structures de transformation. Il ressort des analyses que, des 7 unités ayant participé à l'étude, seules 2 produisent des ananas séchés respectant les normes de 12 à $15 \%$ recommandées en matière de teneur en eau final du produit séché (Rozis, 1995). Par ailleurs, dans les mêmes unités, les teneurs en eau sont différentes d'une production à l'autre et sont régulièrement supérieures à $15 \%$.

\section{pH et acidité totale titrable}

Les $\mathrm{pH}$ des échantillons analysés varient entre 2 et 3 (Tableau 2), et l'acidité totale titrable des échantillons est comprise entre 32 et $70 \mathrm{mEq} / 100 \mathrm{~g}$ soit 2,24 et 4,9 $\mathrm{g} / 100 \mathrm{~g}$ d'échantillon. Dans le groupe J5, les échantillons 1, 2 et 4 présentent les valeurs de $\mathrm{pH}$ similaires mais la différence avec les échantillons 3 et 5 est significative au seuil de $5 \%$. Les unités M1 et T4 produisent des ananas séchés avec des taux d'acidité totale titrable variant très peu ou pas (Tableau 3). 
Quant à la production de B7, les ananas séchés des cinq échantillons se différencient d'une façon marquée par leur acidité. Ces valeurs sont supérieures à $1 \%$, l'acidité maximale qui assure la qualité alimentaire minimale requise par les consommateurs (Marrero et Kader, 2001).

\section{Teneur en extrait sec soluble}

Les taux d'ESS des échantillons d'ananas séchés se situent entre 62 et 73 g/100 $\mathrm{g}$ de produit sec. Ils sont significativement différents au seuil de 5\%, non seulement pour les produits issus d'une même unité de transformation mais également pour les ananas séchés collectés dans les unités différentes (Tableau 4). Les échantillons $\mathrm{n}^{\circ} 1$ de l'unité B7 et $\mathrm{n}^{\circ} 4$ de l'unité J5 présentent respectivement les valeurs les plus faibles et les plus élevées.

\section{Teneur en sucres totaux}

Les composés biodégradables dans la matière organique (surtout les polysaccharides) ont un rôle majeur sur la richesse en sucres de la pulpe. La teneur en sucres totaux représente l'ensemble constitué en solution des matières autres que les sucres. Les valeurs consignées dans le Tableau 5, montrent que la teneur en sucres totaux des tranches séchées varie entre 14,65 et 62,28 $\mathrm{g} / 100 \mathrm{~g}$ de MS respectivement pour les unités T4 et B7. Dans chaque unité, les lots analysés présentent des différences qui sont quelques fois significatives au seuil de $5 \%$. Seules les unités Z6 et M3 ont des échantillons (lots) similaires à quelques exceptions. Pour une même unité, les teneurs en sucres totaux diffèrent significativement au seuil de $5 \%$ du simple au double (cas de l'unité U2 qui possède des échantillons dont les valeurs varient entre 19,86 et 44,55 g/100 $\mathrm{g}$ de matière sèche); du simple au triple d'un lot de production à l'autre (cas de l'unité B7 avec des valeurs de 18,46 et $62,28 \mathrm{~g} / 100 \mathrm{~g}$ de matière sèche).

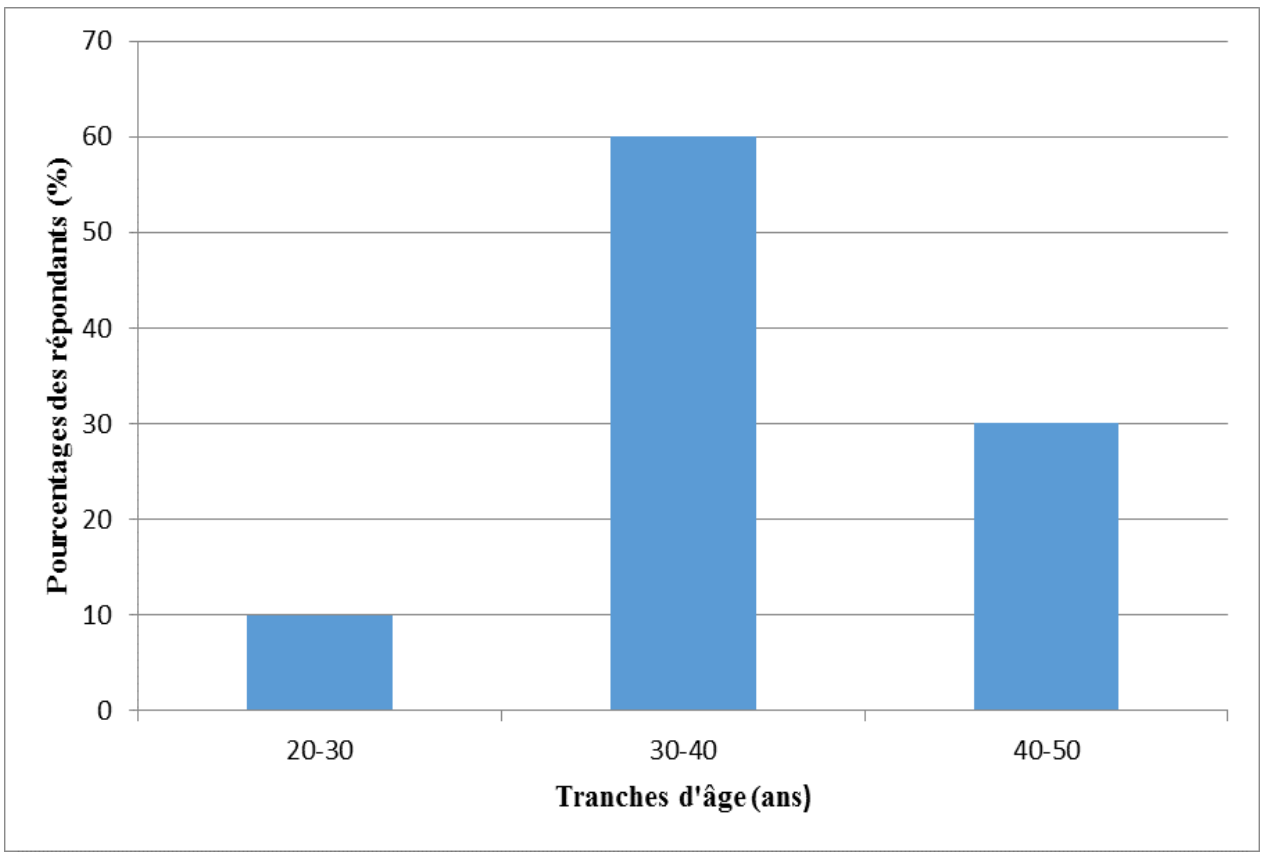

Figure 1 : Les tranches d'âges des transformateurs. 


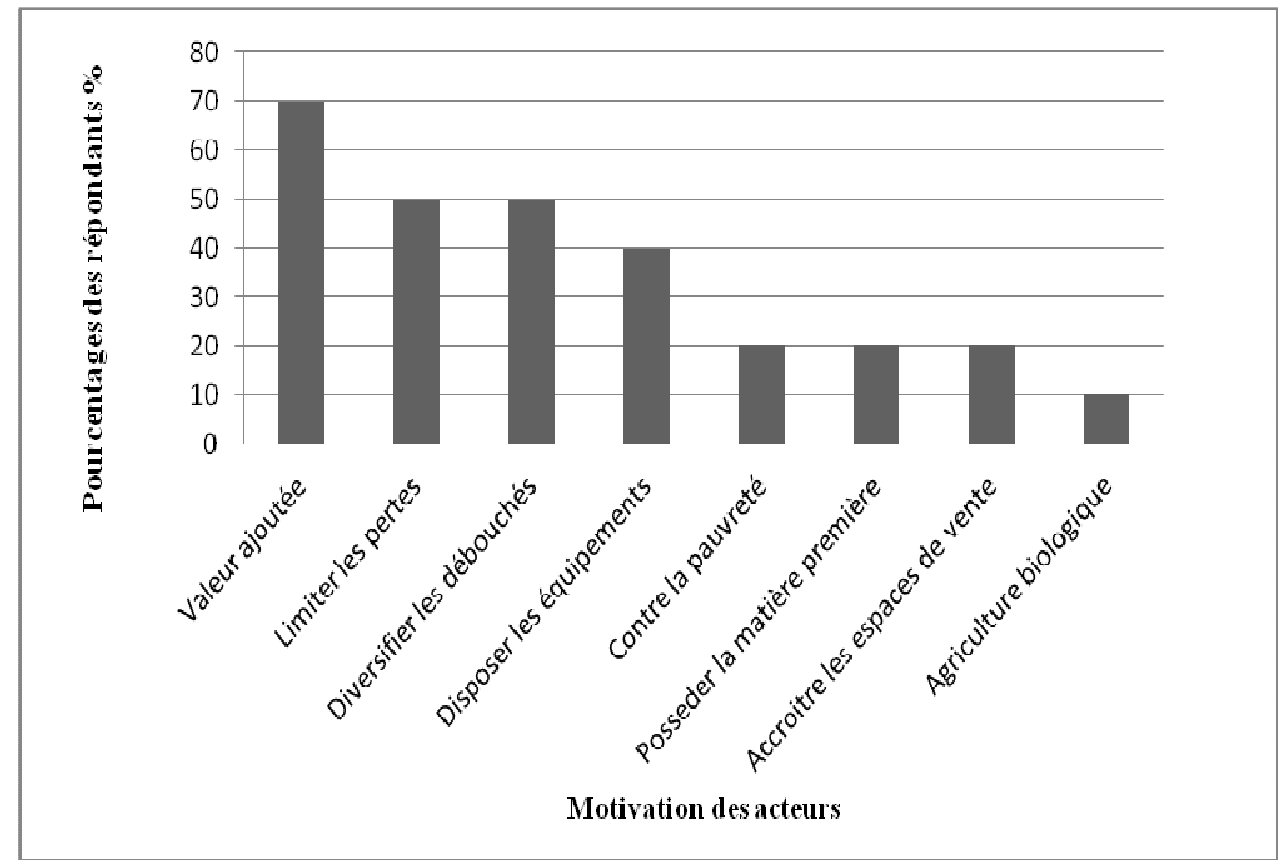

Figure 2 : Les motivations de création des unités de transformation.

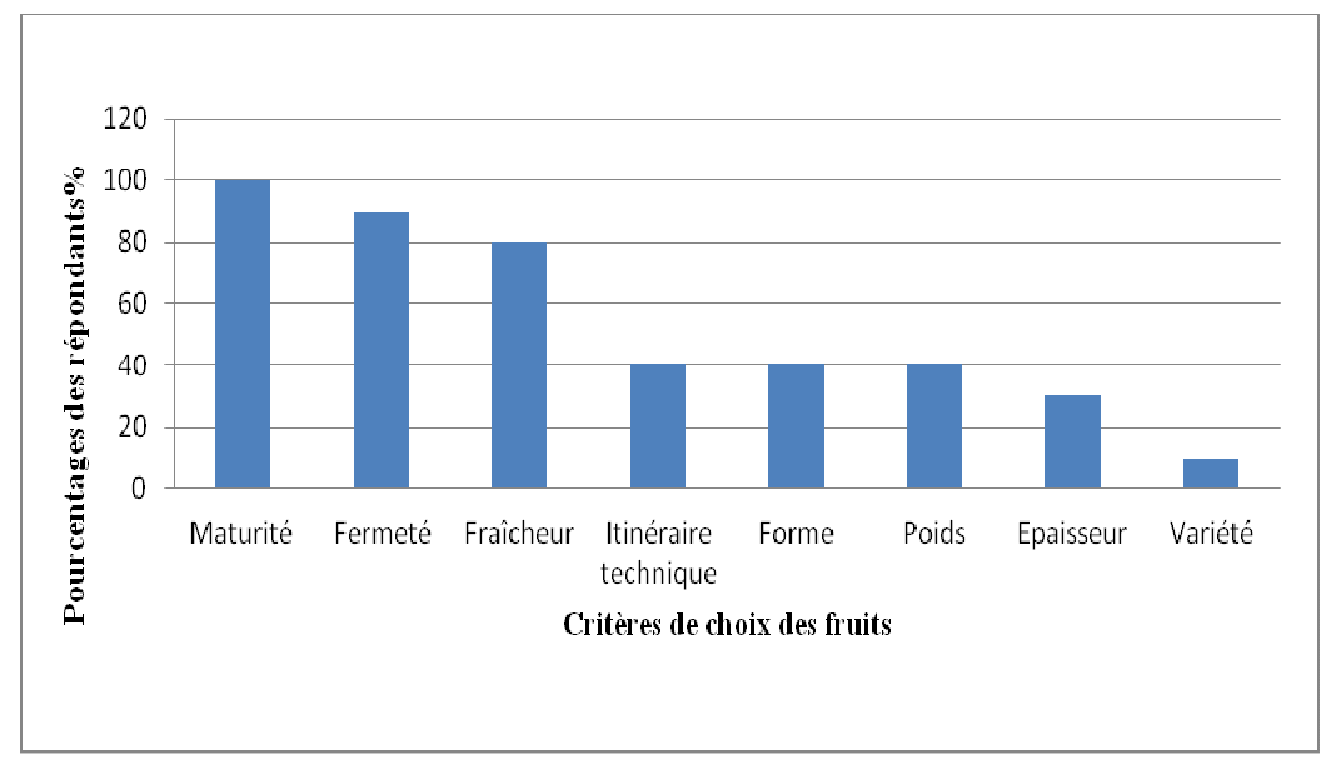

Figure 3 : Les critères de choix de la matière première. 


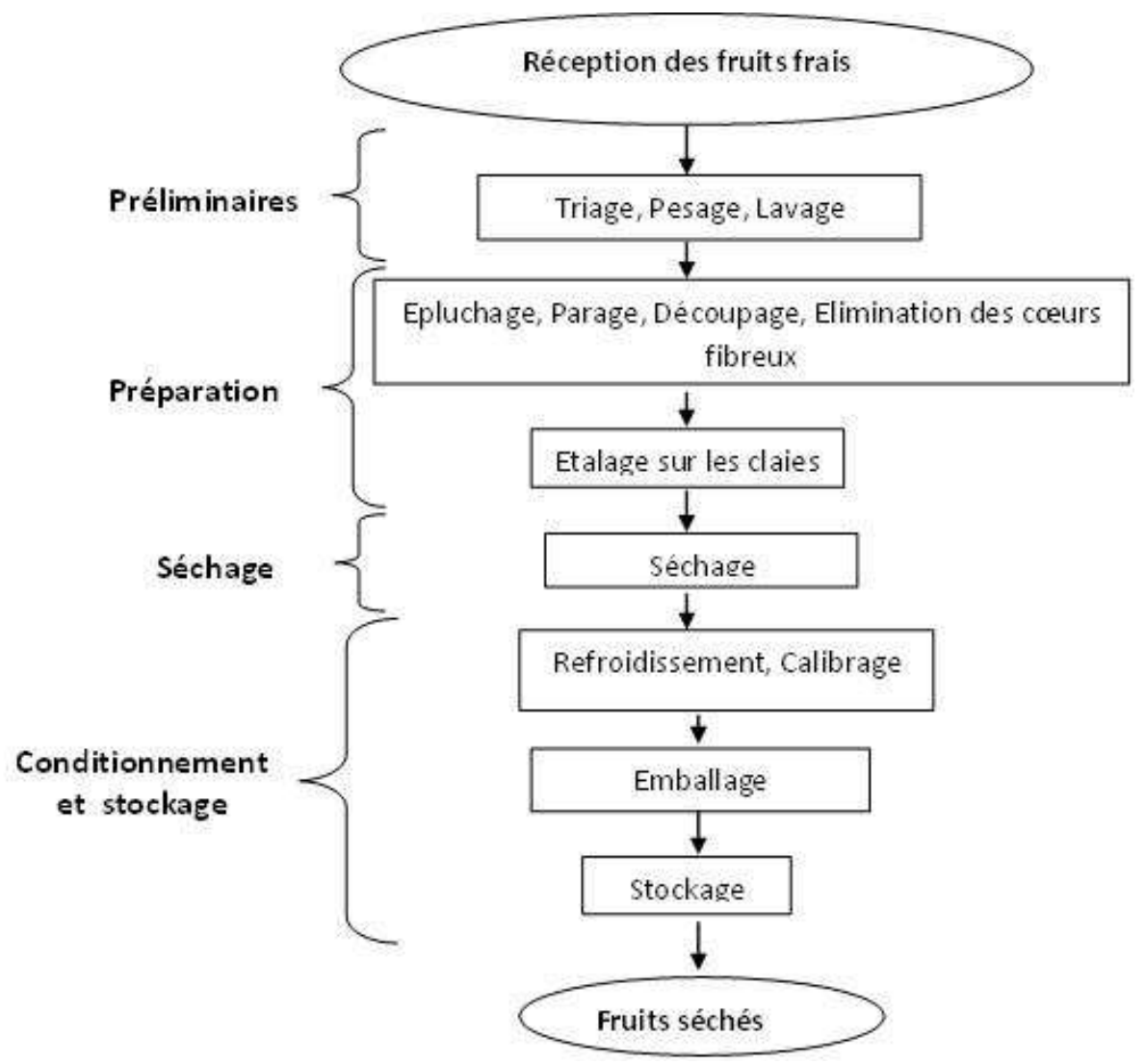

Figure 4: Les principales étapes de la production des ananas séchés dans les unités de transformation.

Tableau 1 : Teneur en eau moyenne (\%) pour chaque échantillon d'ananas séché.

\begin{tabular}{|c|c|c|c|c|c|}
\hline Echantillons & 1 & 2 & 3 & 4 & 5 \\
\hline \multicolumn{6}{|l|}{ Unités } \\
\hline C1* & $14,25 \pm 0,04^{\mathrm{a}}$ & $16,81 \pm 0,32^{b}$ & $15,36 \pm 0,02^{c}$ & $15,81 \pm 0,16^{\mathrm{cd}}$ & $15,52 \pm 0,49^{\mathrm{ce}}$ \\
\hline $\mathbf{U} 2 *$ & $14,53 \pm 0,24^{\mathrm{a}}$ & $15,62 \pm 0,10^{b}$ & $14,53 \pm 0,14^{\mathrm{a}}$ & $14,95 \pm 0,72^{\mathrm{ab}}$ & $14,73 \pm 0,41^{\mathrm{a}}$ \\
\hline M3* & $17,95 \pm 1,52^{\mathrm{abc}}$ & $16,84 \pm 022^{\mathrm{abcd}}$ & $16,83 \pm 0,11^{\mathrm{abcd}}$ & $16,28 \pm 0,04^{\mathrm{bcd}}$ & $15,46 \pm 0,77^{\mathrm{bcd}}$ \\
\hline $\mathbf{T} 4 * *$ & $15,47 \pm 0,18^{\text {abcde }}$ & $15,78 \pm 0,20^{\mathrm{abcd}}$ & $15,47 \pm 0,04^{\text {abcde }}$ & $15,68 \pm 0,25^{\mathrm{abcd}}$ & $15,19 \pm 0,24^{\text {ace }}$ \\
\hline $\mathbf{J 5} * *$ & $17,29 \pm 0,21^{\mathrm{a}}$ & $16,03 \pm 0,08^{\mathrm{a}}$ & $17,16 \pm 0,06^{\mathrm{a}}$ & $13,34 \pm 1,31^{b}$ & $13,97 \pm 1,01^{\mathrm{b}}$ \\
\hline $\mathbf{Z 6} * *$ & $17,51 \pm 0,10^{\mathrm{a}}$ & $18,12 \pm 0,02^{\mathrm{b}}$ & $19,73 \pm 0,06^{\mathrm{c}}$ & $17,50 \pm 0,10^{\mathrm{a}}$ & $16,69 \pm 0,14^{\mathrm{d}}$ \\
\hline B7** & $16,81 \pm 0,49^{\mathrm{abc}}$ & $17,98 \pm 0,03^{\mathrm{ab}}$ & $17,77 \pm 0,88^{\mathrm{ab}}$ & $15,29 \pm 0,44^{\mathrm{ac}}$ & $14,53 \pm 2,43^{\mathrm{ac}}$ \\
\hline
\end{tabular}

Les moyennes ayant les mêmes lettres en exposant dans la même ligne ne sont pas significativement différentes au seuil de $5 \%$.

Les unités portant le caractère $(*)$ sont situées dans le département du Moungo et celles portant $\left(^{* *}\right)$ sont situées dans le département du Wouri. 
Tableau 2: pH moyen des échantillons d'ananas séchés collectés dans les unités de transformation.

\begin{tabular}{|c|c|c|c|c|c|}
\hline Echantillons & 1 & 2 & 3 & 4 & 5 \\
\hline \multicolumn{6}{|l|}{ Unités } \\
\hline C1* & $3,69 \pm 0,54^{\mathrm{a}}$ & $3,73 \pm 0,52^{\mathrm{a}}$ & $3,71 \pm 057^{\mathrm{a}}$ & $3,98 \pm 0,09^{a}$ & $3,94 \pm 0,11^{\mathrm{a}}$ \\
\hline $\begin{array}{l}\text { U2* } \\
\text { M3* }\end{array}$ & $\begin{array}{c}3,63 \pm 0,45^{\mathrm{a}} \\
3,51 \pm 0,43^{\text {abce }}\end{array}$ & $\begin{array}{c}3,61 \pm 0,60^{\mathrm{a}} \\
3,97 \pm 0,02^{\mathrm{abcde}}\end{array}$ & $\begin{array}{c}3,95 \pm 0,12^{\mathrm{a}} \\
3,86 \pm 0,40^{\mathrm{abcde}}\end{array}$ & $\begin{array}{c}3,43 \pm 0,45^{\mathrm{a}} \\
4,00 \pm 0,03^{\mathrm{bcde}}\end{array}$ & $\begin{array}{c}3,65 \pm 0,49^{\mathrm{a}} \\
3,96 \pm 0,13^{\mathrm{abcde}}\end{array}$ \\
\hline $\mathbf{T} 4 * *$ & $3,77 \pm 0,01^{\mathrm{abd}}$ & $3,39 \pm 0,01^{\mathrm{abcd}}$ & $3,10 \pm 0,01^{b c}$ & $3,60 \pm 0,45^{\mathrm{abd}}$ & $3,99 \pm 0,10^{\text {ae }}$ \\
\hline $\mathbf{J 5}$ ** & $3,56 \pm 0,01^{\mathrm{a}}$ & $3,48 \pm 0,00^{\mathrm{a}}$ & $3,75 \pm 0,02^{\mathrm{a}}$ & $3,54 \pm 0,10^{b}$ & $3,88 \pm 0,13^{b}$ \\
\hline $\mathrm{Z6} * *$ & $3,67 \pm 0,01^{\mathrm{a}}$ & $3,89 \pm 0,00^{\mathrm{b}}$ & $3,82 \pm 0,03^{b}$ & $3,83 \pm 0,07^{\mathrm{b}}$ & $3,60 \pm 0,13^{\mathrm{a}}$ \\
\hline B 7** & $3,84 \pm 0,31^{\mathrm{a}}$ & $3,53 \pm 0,27^{\mathrm{a}}$ & $3,85 \pm 0,18^{\mathrm{a}}$ & $3,84 \pm 0,17^{\mathrm{a}}$ & $3,47 \pm 0,44^{\mathrm{a}}$ \\
\hline
\end{tabular}

Les moyennes ayant les mêmes lettres en exposant dans la même ligne ne sont pas significativement différentes au seuil de $5 \%$.

Les unités portant le caractère $(*)$ sont situées dans le département du Moungo et celles portant $\left(^{* *}\right)$ sont situées dans le département du Wouri.

Tableau 3: Acidité totale titrable ( $\mathrm{mEq} / 100 \mathrm{~g}$ des échantillons d'ananas séché) des différents échantillons collectés dans les unités de transformation de la région du Littoral Cameroun.

\begin{tabular}{|c|c|c|c|c|c|}
\hline$\frac{\text { Echantillons }}{\text { Unités }}$ & 1 & 2 & 3 & 4 & 5 \\
\hline C1* & $42,91 \pm 0,73^{\mathrm{a}}$ & $42,18 \pm 0,00^{\mathrm{a}}$ & $54,03 \pm 1,1^{b}$ & $60,85 \pm 1,83^{c}$ & $56,48 \pm 1,51^{b}$ \\
\hline $\mathbf{U} 2 *$ & $32,48 \pm 0,42^{b}$ & $41,21 \pm 1,11^{\mathrm{a}}$ & $36,85 \pm 0,42^{\mathrm{c}}$ & $41,94 \pm 2,75^{\mathrm{a}}$ & $40,73 \pm 0,73^{\mathrm{a}}$ \\
\hline M3* & $40,00 \pm 0,00^{\mathrm{a}}$ & $36,22 \pm 0,73^{\mathrm{ac}}$ & $39,52 \pm 0,42^{\text {ad }}$ & $40,48 \pm 6,72^{\mathrm{ae}}$ & $54,79 \pm 3,73^{\mathrm{b}}$ \\
\hline $\mathbf{T} 4 * *$ & $57,21 \pm 2,94^{\mathrm{a}}$ & $66,91 \pm 11,43^{b}$ & $70,79 \pm 16,10^{\mathrm{c}}$ & $61,58 \pm 0,84^{\mathrm{d}}$ & $58,42 \pm 2,22^{\mathrm{e}}$ \\
\hline $\mathbf{J 5} * *$ & $49,45 \pm 0,00^{\mathrm{ab}}$ & $50,18 \pm 0,73^{\mathrm{abc}}$ & $48,97 \pm 0,42^{\mathrm{ab}}$ & $59,64 \pm 1,26^{\mathrm{d}}$ & $52,36 \pm 3,33^{b c}$ \\
\hline Z6*** & $54,06 \pm 0,042^{\mathrm{a}}$ & $54,06 \pm 3,59^{\mathrm{a}}$ & $51,88 \pm 0,42^{\mathrm{a}}$ & $77,82 \pm 0,73^{b}$ & $54,30 \pm 1,83^{\mathrm{a}}$ \\
\hline $\mathbf{B} 7 * *$ & $40,73 \pm 0,73^{\mathrm{a}}$ & $43,64 \pm 0,73^{b}$ & $42,18 \pm 0,73^{\mathrm{c}}$ & $40,24 \pm 0,42^{\mathrm{a}}$ & $38,55 \pm 0,00^{\mathrm{d}}$ \\
\hline
\end{tabular}

Les moyennes ayant les mêmes lettres en exposant dans la même ligne ne sont pas significativement différentes au seuil de $5 \%$.

Les unités portant le caractère $(*)$ sont situées dans le département du Moungo et celles portant $\left(^{* *}\right)$ sont situées dans le département du Wouri.

Tableau 4 : Taux d'extrait sec soluble en g/100 g des échantillons d'ananas séchés.

\begin{tabular}{|c|c|c|c|c|c|}
\hline Echantillons & 1 & 2 & 3 & 4 & 5 \\
\hline \multicolumn{6}{|l|}{ Unités } \\
\hline C1* & $71,60 \pm 0,69^{\mathrm{a}}$ & $69,20 \pm 0,69^{b}$ & $66,40 \pm 0,69^{c}$ & $68,40 \pm 1,20^{b}$ & $66,40 \pm 0,69^{c}$ \\
\hline $\mathbf{U} 2 *$ & $69,20 \pm 3,02^{\mathrm{a}}$ & $65,60 \pm 1,83^{b}$ & $72,40 \pm 0,69^{\mathrm{c}}$ & $70,00 \pm 0,69^{\mathrm{ac}}$ & $67,60 \pm 0,69^{\mathrm{ab}}$ \\
\hline M3* & $64,00 \pm 0,69^{\mathrm{a}}$ & $71,60 \pm 0,69^{\mathrm{b}}$ & $65,20 \pm 0,69^{\mathrm{ac}}$ & $66,00 \pm 1,20^{\mathrm{ac}}$ & $67,60 \pm 0,69^{c}$ \\
\hline $\mathbf{T} 4 * *$ & $66,40 \pm 0,69^{a}$ & $69,20 \pm 0,69^{\mathrm{b}}$ & $63,60 \pm 1,20^{\mathrm{c}}$ & $71,20 \pm 0,69^{\mathrm{d}}$ & $66,80 \pm 0,69^{\mathrm{a}}$ \\
\hline $\mathbf{J 5} * *$ & $72,40 \pm 0,69^{\mathrm{a}}$ & $68,80 \pm 0,69^{b}$ & $66,40 \pm 1,83^{\mathrm{b}}$ & $73,60 \pm 1,83^{\mathrm{a}}$ & $68,00 \pm 0,69^{b}$ \\
\hline $\mathbf{Z 6} \mathbf{6}^{* *}$ & $64,00 \pm 0,69^{\mathrm{abcd}}$ & $62,80 \pm$ & $63,60 \pm 1,20^{\mathrm{abcde}}$ & $68,00 \pm 3,02^{\text {acde }}$ & $68,40 \pm 3,17^{\text {acde }}$ \\
\hline $\mathbf{B} 7 * *$ & $62,40 \pm 2,40^{\mathrm{a}}$ & $65,60 \pm 0,69^{b}$ & $71,20 \pm 0,69^{c}$ & $71,20 \pm 1,39^{\mathrm{c}}$ & $73,20 \pm 2,08^{\mathrm{d}}$ \\
\hline
\end{tabular}


Tableau 5 : Teneur en sucres totaux (g/100 g de MS) des échantillons d'ananas collectés.

\begin{tabular}{cccccc}
\cline { 1 - 5 } Echantillons & $\mathbf{1}$ & $\mathbf{2}$ & $\mathbf{3}$ & $\mathbf{4}$ & $\mathbf{5}$ \\
\cline { 1 - 5 } Unités & & & & & \\
\cline { 1 - 5 } $\mathbf{C 1}^{*}$ & $46,54 \pm 4,26^{\mathrm{a}}$ & $45,76 \pm 4,30^{\mathrm{ac}}$ & $27,64 \pm 7,51^{\mathrm{b}}$ & $33,24 \pm 1,03^{\mathrm{bc}}$ & $37,10 \pm 1,05^{\mathrm{c}}$ \\
$\mathbf{U 2}^{*}$ & $44,55 \pm 4,36^{\mathrm{a}}$ & $27,72 \pm 4,34^{\mathrm{b}}$ & $19,86 \pm 4,95^{\mathrm{c}}$ & $24,27 \pm 1,92^{\mathrm{bc}}$ & $38,20 \pm 2,17^{\mathrm{a}}$ \\
$\mathbf{M 3}^{*}$ & $27.39 \pm 4.32^{\mathrm{a}}$ & $19.31 \pm 1.06^{\mathrm{b}}$ & $27.03 \pm 5.76^{\mathrm{ab}}$ & $21.37 \pm 1.10^{\mathrm{ab}}$ & $27.69 \pm 4.55^{\mathrm{a}}$ \\
$\mathbf{T 4}^{* *}$ & $14,65 \pm 2,16^{\mathrm{a}}$ & $34,31 \pm 2,81^{\mathrm{b}}$ & $21,16 \pm 3,26^{\mathrm{a}}$ & $28,83 \pm 4,28^{\mathrm{b}}$ & $18,94 \pm 3,94^{\mathrm{a}}$ \\
$\mathbf{J 5}^{* *}$ & $31,61 \pm 4,03^{\mathrm{a}}$ & $31,14 \pm 2,89^{\mathrm{ab}}$ & $35,99 \pm 3,30^{\mathrm{abc}}$ & $37,57 \pm 2,99^{\mathrm{c}}$ & $36,79 \pm 2,07^{\mathrm{ac}}$ \\
$\mathbf{Z 6}^{* *}$ & $21,69 \pm 4,43^{\mathrm{a}}$ & $20,73 \pm 4,03^{\mathrm{a}}$ & $21,14 \pm 4,98^{\mathrm{a}}$ & $25,02 \pm 2,25^{\mathrm{a}}$ & $34,69 \pm 2,85^{\mathrm{b}}$ \\
$\mathbf{B 7}^{* *}$ & $33,65 \pm 10,73^{\mathrm{a}}$ & $18,46 \pm 1,12^{\mathrm{b}}$ & $19,54 \pm 2,15^{\mathrm{b}}$ & $62,28 \pm 2,13^{\mathrm{c}}$ & $56,98 \pm 8,68^{\mathrm{c}}$ \\
\hline
\end{tabular}

Les moyennes ayant les mêmes lettres en exposant dans la même ligne ne sont pas significativement différentes au seuil de $5 \%$.

Les unités portant le caractère (*) sont situées dans le département du Moungo et celles portant (**) sont situées dans le département du Wouri.

\section{DISCUSSION}

Le nombre d'employés rencontrés dans les dix unités de séchage conduit à un regroupement en deux catégories. Selon Fellows et Axtell (2005) et en fonction du nombre de personnes travaillant dans ces structures, on a 5 micros entreprises qui emploient entre 2 et 4 personnes (moins de 5 personnes) et 5 petites entreprises qui utilisent un effectif variant entre 5 et 15 personnes. De plus, la fréquence de production d'ananas séché est fortement liée aux commandes. Cette situation serait due aux problèmes divers rencontrés dans les structures notamment l'absence des circuits de distribution des fruits séchés. La faible présence des jeunes dans le secteur du séchage de l'ananas pourrait être due au manque de moyens matériels et financiers pour le démarrage d'une telle activité malgré la maîtrise de la technique de séchage. En effet, les responsables de ces structures ont un niveau d'instruction allant du primaire $(30 \%)$ au supérieur $(40 \%)$ avec un taux de $30 \%$ pour le secondaire; ce qui leur permettrait de mieux assimiler les technologies relatives au séchage des fruits et légumes. Les unités ont été créées pour diverses raisons, toutefois, 3 raisons principales présentées par les acteurs indiquent la situation actuelle de l'ananas et des fruits en général. En effet, la production et les échanges commerciaux des fruits tropicaux ont augmenté au cours de la dernière décennie, bien qu'il soit difficile de quantifier le volume, celui-ci est relativement élevé selon Temple (2001). Parmi ces fruits tropicaux, l'ananas occupe après la banane, la deuxième place des fruits à l'exportation vers les marchés de l'Union Européenne (AGROCOM, 2002). Sa production est estimée à environ 35 tonnes /ha selon les statistiques de la FAO en 2014. Le séchage permet aux acteurs de conserver pendant longtemps le fruit et de créer de la valeur ajoutée. Malheureusement, les acteurs ne sont pas capables de justifier les critères de choix de la matière première. Toutefois, l'impact du critère choisi sur la qualité du produit fini est plus ou moins connu car le goût de l'ananas s'exprime habituellement sous forme d'une combinaison des principes sucré et acide qui donnent une indication du degré de maturité et de comestibilité de la pulpe. Il a été démontré en 2004 par les travaux de Berüter, cité par Le Mire (2012) que le potassium joue un rôle important dans l'accumulation des acides organiques dans de nombreux fruits et exerce une influence particulière sur la qualité organoleptique de ces fruits.

Dans le cadre de ce diagnostic, la maturité des fruits est d'une manière générale choisie sur la base de la coloration de 
l'épiderme sans mesures ni contrôles de certaines caractéristiques physicochimiques, ce qui indique que le degré réel de maturité des ananas n'est pas connu des transformateurs. Les locaux utilisés ne répondent pas toujours aux standards destinés à la transformation, et sont très souvent implantés sur des sites inappropriés. En effet, la majorité des structures utilise les ateliers et/ou hangars des domiciles comme locaux de séchage qui requièrent une salle de réception, de lavage, d'épluchage, de pesage, de séchage, de conditionnement et de conservation/stockage. Bien plus, le découpage manuel des fruits donne des épaisseurs irrégulières des tranches et conduit à un séchage hétérogène des fruits. En plus de la qualité initiale du fruit, la taille des morceaux séchés est un facteur qui conditionne la qualité des produits séchés dans la transformation (Hilaire, 2008).

$\mathrm{Au}$ terme $\mathrm{du}$ processus, aucun instrument de mesure n'est utilisé pour le contrôle de la teneur en eau finale, qui est un critère très déterminant non seulement pour la qualité mais également pour la durée de conservation du produit fini afin d'éviter des cas de contamination fongique. Par ailleurs, la détermination des caractéristiques physicochimiques permet de ressortir la qualité des échantillons à la fin de chaque production. Le problème de ravitaillement en eau potable se pose également dans certaines unités. Bien que toutes ces tâches soient effectuées manuellement, les acteurs rencontrés sont plus ou moins informés de bonnes pratiques d'hygiène qu'ils essayent autant que faire ce peu d'appliquer afin de limiter les risques de contamination par les microorganismes et les pertes par dégradation du fruit débarrassé de sa peau. En plus du contrôle des paramètres de séchage (temps et température) et de fin de l'opération qui sont effectués selon l'expérience professionnelle de l'acteur et de la qualité du séchoir utilisé, les difficultés ont été perçues au niveau du chargement des claies, la permutation des claies et la ventilation du séchoir. Les claies sont chargées au fur et à mesure que le découpage est effectué et placées dans les séchoirs préchauffés à $75^{\circ} \mathrm{C}$. Pendant $18 \mathrm{~h}$ à $36 \mathrm{~h}$, à la température de séchage variant entre $65{ }^{\circ} \mathrm{C}$ et $70{ }^{\circ} \mathrm{C}$, l'air chaud qui traverse les tranches d'ananas est transféré au produit par convection tandis que l'eau est enlevée par entraînement grâce à l'air sec qui arrive par le compartiment inférieur. Compte tenu du fait que la performance d'un séchoir et la qualité de l'opération dépendent des quantités déposées sur chaque claie, il serait nécessaire de respecter la densité de chargement au cours du séchage pour obtenir un produit uniforme de qualité. Dans la plupart des unités visitées, l'emballage polyéthylène à basse densité domine très largement dans le conditionnement des fruits séchés. Pour son imperméabilité à l'humidité et sa soudabilité thermique, les fabricants nationaux d'emballage polyéthylène ne donnent pas toujours satisfaction aux transformateurs qui rencontrent d'énormes problèmes pendant le conditionnement des tranches séchées dans leurs structures respectives. Pour lever cette contrainte au niveau du marché international, certains acteurs utilisent des sachets identiques provenant de leurs partenaires européens. Les autres sécheurs ont fait de gros efforts dans l'amélioration de leurs sachets, malgré la présence des sachets inadéquats sur le marché national, ils mettent les moyens pour avoir les matières plastiques pouvant tenir à la fermeture et conserver au mieux les fruits séchés. La commercialisation des fruits séchés rencontre plusieurs obstacles parmi lesquels : l'abondance des fruits frais dans les différentes localités du Cameroun; les habitudes alimentaires des populations locales qui freinent l'écoulement des fruits séchés; l'existence d'une concurrence locale rude entre le marché des produits séchés et le marché de produits frais.

La teneur en eau est fortement corrélée à la notion d'activité de l'eau. La notion d'activité biologique de l'eau est primordiale en alimentation puisqu'elle permet de mettre en œuvre une stratégie de protection des aliments en contrôlant les détériorations physico-chimiques, les activités enzymatiques 
et la multiplication des populations microbiennes. En l'absence des moyens et d'équipement pour l'évaluation de l'activité de l'eau des produits séchés prélevés dans les unités de transformations enquêtées, seules leurs teneurs en eau ont été déterminées. Les valeurs moyennes enregistrées se situent entre 13,34 et $19,73 \%$ et varient en fonction des échantillons dans les unités. Dans la plupart des cas, les teneurs en eau sont relativement plus élevées comparativement aux valeurs recommandées situées entre 12 et $15 \%$ (Tableau 1). Par conséquent, la qualité tant au niveau de la conservation que de la consommation sera moindre et se traduirait par la diminution de la durée de stockage comme l'indique Agassounon et al. (2012) pour la tomate. La variabilité observée sur les échantillons montre que les systèmes ne sont pas stables dans la production des fruits séchés et cela impliquerait que toute négligence des conditions de travail pourrait entraîner l'augmentation de l'humidité résiduelle dans les tranches séchées avant la vente complète de tous les produits. Les valeurs $\mathrm{du} \mathrm{pH}$ confirment le caractère acide des tranches d'ananas séchés (Tableau 2). La détermination $\mathrm{du} \quad \mathrm{pH}$ fournit des renseignements sur le degré de dissociation des acides présents dans le fruit. En général, le pH reste inférieur à 4,5 c'est-à-dire correspond à des milieux acides. L'acidité totale titrable des échantillons est comprise entre 32 et 70 $\mathrm{mEq} / 100 \mathrm{~g}$ soit 2,24 et $4,9 \mathrm{~g} / 100 \mathrm{~g}$ d'échantillon (Tableau 3). Ces valeurs sont supérieures à $1 \%$ qui est l'acidité maximale qui assure la qualité alimentaire minimale requise par les consommateurs (Morrero et Kader, 2006). De même, nous constatons que l'extrait sec soluble des produits analysés ne se trouve pas dans la norme de $12{ }^{\circ}$ Brix recommandée par le codex alimentarius pour les ananas. Toutes les valeurs sont inférieures à $72 \mathrm{~g} / 100 \mathrm{~g}$ qui est la valeur minimale d'extrait sec soluble acceptable dans l'ananas à la récolte (Tableau 4). Les ananas sont des fruits non-climactériques et doivent donc être récoltés quand ils sont prêts à la consommation. En effet, la qualité de l'ananas séché dépend largement des propriétés des fruits frais utilisés dans sa fabrication. Les résultats des paramètres physicochimiques obtenus seraient liés à l'état nutritionnel de la plante sous l'effet de la fertilisation combinée à l'action du climat et des itinéraires techniques. Ce genre de relation influence de la matière première sur la qualité du produit final a été observé par de nombreux auteurs (Hunsche et al., 2003 ; Lima et al., 2008 ; Lester et al., 2010 ; Yasin et al., 2010 ; Quaggio, 2011) cités par Le Mire (2012). Le potassium semble en effet impliqué dans l'équilibre des charges des acides organiques dans de nombreux fruits comme c'est le cas de la banane (Le Mire, 2012). En effet l'incidence la plus nette de l'apport du potassium s'exercerait sur l'extrait sec et l'acidité des fruits qui augmentent avec les doses appliquées à la plante; le potassium aide à l'amélioration du parfum et la saveur des fruits. Cependant, la qualité des fruits séchés dépend en partie du degré de maturité physiologique souhaitée (Hilaire, 2008). L'acidité varie quelquefois significativement ( $\mathrm{p}<0,05)$ d'une part entre les échantillons collectés dans la même unité de séchage et d'autre part entre les produits finis collectés dans les différentes unités (Tableau 3). Toutefois, le facteur acidité totale titrable pourrait servir d'indicateur du degré de maturité des fruits qui sont traités dans les différentes unités de séchage. Les teneurs en sucre et en acides organiques sont des caractères prioritaires dans la détermination de la qualité organoleptique. Cependant, ces composés sont inégalement répartis dans l'ananas car sa structure est hétérogène; la partie supérieure du fruit est toujours moins sucrée et plus acide que celle inférieure quel que soit le niveau de maturité du fruit. La teneur en sucres totaux semble être celui présentant le plus de variation (Tableau 5). L'analyse du tableau permet de constater que les teneurs en sucre total des tranches séchées malgré quelques variabilités au niveau des échantillons, sont inférieures au taux d'extrait sec soluble. Ces résultats pourraient s'expliquer par les différents lieux 
d'approvisionnement en fruits frais pour chaque production ou par l'itinéraire technique de production des fruits utilisés dans les unités. En tenant compte des travaux d'Hilaire (2008) sur le séchage de deux variétés de mangues, la faible teneur en sucres totaux pourrait également être due à une consommation importante des microorganismes en sucre.

\section{Conclusion}

La caractérisation des unités de séchage et des produits dérivés dans la région du Littoral du Cameroun, a permis d'identifier une dizaine d'unités de production d'ananas séchés, et de comprendre les variations des paramètres physicochimiques des lots issus des différentes unités de transformation. Elle a permis de conclure qu'en général les procédures de séchage sont soit non standardisées, soit méconnues des opérateurs, soit non maîtrisées par les acteurs intervenant dans la chaine de production d'ananas séchés dans la région du Littoral - Cameroun. Pour éviter que les ananas séchés produits dans les unités de séchage soient de mauvaise qualité tant au niveau de la conservation que de la consommation, il serait souhaitable d'optimiser un protocole standard de séchage d'ananas à l'aide d'équipements semiartisanaux, de renforcer les capacités techniques des acteurs de la filière de séchage d'ananas au Cameroun à travers des formations et des sessions de recyclage. Aussi, il serait important que chaque unité possède un kit d'équipement minimal pour l'évaluation de quelques critères physicochimiques devant leur permettre de contrôler la qualité de leurs productions. Par ailleurs, il serait indispensable de poursuivre cette étude par un programme qui prendra en compte l'évaluation des qualités microbiologiques des ananas séchés prêts à la consommation, et leur durée de conservation ou de stockage à température ambiante.

\section{CONFLIT D'INTERETS}

Les auteurs déclarent qu'ils n'ont aucun conflit d'intérêts.

\section{CONTRIBUTIONS DES AUTEURS}

NBMN a réalisé les enquêtes et collecté les échantillons d'ananas séchés. Elle a participé à l'évaluation des paramètres physicochimiques et à la rédaction du manuscrit. NNG a réalisé les analyses physicochimiques au laboratoire de Technologie Post-Récolte du CARBAP et a activement participé à la rédaction et à l'édition du manuscrit. NR a supervisé l'ensemble des travaux et a participé la rédaction et à l'édition du manuscrit. Tous les auteurs ont lu et approuvé le manuscrit.

\section{REMERCIEMENTS}

Les auteurs remercient l'institut de Recherche Agricole pour le Développement (IRAD) et l'Université de Ngaoundéré. Leur gratitude va également à l'endroit du Centre Africain de Recherches sur Bananiers et Plantains (CARBAP) pour les moyens consentis lors de la mise en œuvre de cette étude et de son personnel pour sa disponibilité.

\section{REFERENCES}

AFNOR (Association Française de Normalisation). 1981. Recueil des Normes Françaises, Corps Gras, Graines Oléagineuses, Produits Dérivés ( $2^{\text {ème }}$ édition). France Paris ; 438 p.

AFNOR (Association Française de Normalisation). 1982. Recueil des Normes Françaises des Produits Dérivés des Fruits et Légumes. Jus de Fruits (Première édition). Paris de la défense, France; 327 p.

Agassounon DTM, Gomez S, Tchobo FP, Soumanou MM, Toukourou F. 2012. Essai de conservation de la tomate par la technique de la déshydratation imprégnation par immersion (DII). Int. J. Biol. Chem. Sci., 6(2): 657-669.

AGROCOM (Agriculture, Agro-industrie, Communication). 2002. Guide de production et de protection de l'ananas. PDE-Cameroun. Les publications d'AGROCOM, 38p 
Dadzie BK, Orchard JE. 1997. Evaluation post récolte des hybrides de bananiers et bananiers plantain: critères et méthodes. Guides Technique Inibap, 26-30.

Dubois M, Gilles KA, Hamilton JK, Robert P, Smith F. 1965. Colorimetric methods for micro determination of sugar and related substances. Anal. Chem., 72(6): 953-956.

Fellows PJ, Axtell B. 2005. Créer et Gérer une Petite Entreprise Agroalimentaire. CTA/GRET: 280p.

Hamadé S. 2003. Le commerce des produits agricoles dans les pays ACP et son rôle dans la dynamisation des économies locales: le cas de la filière fruits et légumes. Rapport de l'Institut de l'Environnement et de Recherches Agricoles au sein de la Cellule Agroéconomique du CRREA.

Hilaire JU. 2008. Etude comparative de deux (2) méthodes de séchage (séchoir solaire, Etuve) dans la transformation de deux variétés de mangues. Mémoire du diplôme d'Ingénieur Agronome, Un UEH. $44 \mathrm{p}$

Le Mire G. 2012. Etude de l'impact d'une fertilisation potassique sur les caractéristiques physicochimiques de la banane dessert au cours de sa croissance et à maturité. Memoire de Stage de fin d'études, Master FAGE, Univ. Lorraine, 32p

Marrero A, Kader A. 2006. Quality changes and nutrient retention in fresh-cut versus whole fruits during storage. $J$. Agric. Food Chem., 54: 4284 - 4296.

Monkam N. 2003. Le marché régional des fruits et légumes : potentialités et contraintes. Communication AGROPME Ingénierie; Séminaire sur « La qualité et les procédés des fruits et légumes séchés » du 3 au 5 Décembre à Douala, Cameroun, Pp 2 - 11 .

Ndindeng S. 2005. Diagnostic des méthodes de récolte, de conservation et de transformation des fruits locaux. Rapport annuel de l'IRAD (Institut de Recherche Agricole pour le Développement), Yaoundé Cameroun, 77p.

Rozis JF. 1995. Sécher les Produits Alimentaires: Techniques, Procédés, Equipements. GRET, Ministère de la coopération, CTA: France, Paris; 344p.

Tchango Tchango J. 2003. Le séchage de la banane: Approche qualité et séchage conventionnel. Communication du CARBAP. Séminaire sur «La qualité et les procédés des fruits et légume séchés » du 3 au 5 Décembre à Douala, Cameroun ; Pp 1 - 13.

Temple L. 2001. Quantification des productions et échanges de fruits et légumes au Cameroun. Cahiers Agricultures, 10: 87-94.

Tossou CC, Capo-chichi DBE, Yedomonhan H. 2012. Diversité et caractérisation morphologique des variétés d'ananas (Ananas comosus (L.) Merrill) cultivées au Bénin. J. Appl. Biosci., 87: 8113-8120.

FAOSTAT. 2014. Production of pineapple in Cameroon in 2012. http://faostat.fao.org/site/567/DesktopD efault.aspx?PageID=567\#ancor. Consulté le 20 octobre 2014.

Brat P, Hoang LN, Soler A, Reynes M, Brillouet JM. 2004. Physicochemical characterization of a new pineapple hybrid. (Flhoran41 Cv.). J. Agric. Food Chem., 52(20): 6170-6177. 